\title{
Effect of High Pressure Torsion on corrosion behavior of High Entropy Alloy in alkaline solution
}

\author{
Zhiwei Song a, Yonghao Zhao b , Guanzhong Wu, Xuzhou Gao
}

School of Materials Science and Engineering, Nanjing University of Science and Technology, Nanjing 210094, China

aclrszw30@163.com, byhzhao@njust.edu.cn

Keywords: High entropy alloy, high pressure torsion, corrosion behavior, passivation.

\begin{abstract}
The high pressure torsion (HPT) process was used to process the FeNiCoMoV high-entropy alloy. The open-circuit potential test, the potentiostatic polarization curve test and the electrochemical impedance spectroscopy were used to study the effect of corrosion behavior of FeNiCoMoV High Entropy Alloy in $1 \mathrm{M} \mathrm{NaOH}$ Solution. The results show that the grain refinement of FeNiCoMoV alloy is significant after 6 cycles of high pressure torsion, the phase structure has not changed and the electrochemical impedance of HPT has a significant increase in $1 \mathrm{M} \mathrm{NaOH}$ solution. The potential is $11.97 \%$ higher than the initial state, the corrosion current density is $93.77 \%$ lower than the initial state, and the corrosion resistance is improved obviously.
\end{abstract}

\section{Introduction}

High Entropy Alloy [1] (HEA) is a new type of alloy whose number of main elements $\mathrm{n}$ is 5 to 13 and the mass fraction of each main element is between 5\% and 35\%. High entropy alloys tend to form simple solid solution structures without the appearance of complex intermetallic compounds and exhibit high strength, high hardness, thermal stability [2] and corrosion resistance and other characteristics, with a wide range of applications, corrosion performance research has become a hot topic. However, the current research focuses on the relationship between coarse-grained high-entropy alloy composition, organizational structure and corrosion resistance [3], and less involves ultra-fine grain or nanocrystalline high entropy alloys.

However, when the grain size of the high entropy alloy is refined to the nanometer level, it will inevitably lead to its corrosion resistance and coarse grain high entropy alloy is different, however, the current on the nano-crystal high entropy alloy corrosion resistance is still fresh it was reported [4]. Extreme plastic deformation has been widely used in the refinement of conventional metal materials to prepare bulk ultrafine or nanocrystalline materials, resulting in excellent mechanical properties. High pressure torsion [5] can maintain the full size of the sample and can efficiently introduce large strain to control the microstructure of the deformed sample, which has become the most popular method of severe plastic deformation. Therefore, in this paper, FeNiCoMoV high entropy alloy as the research object, through the high-pressure reverse (HPT) technology to grain size to nano-level, and then studied its $1 \mathrm{M} \mathrm{NaOH}$ solution in the electrochemical properties, and the coarse - grained high - entropy alloys were compared and analyzed.

\section{Experiment}

\subsection{Sample preparation}

The initial state alloy used in this experiment is to use high purity $\mathrm{Fe}, \mathrm{Ni}, \mathrm{Co}, \mathrm{Mo}, \mathrm{V}$ as raw materials, under the protection of high purity argon vacuum arc melting method used to prepare high entropy alloy button ingots, each alloy button ingot at high temperature $1200^{\circ} \mathrm{C}$ smelting 3 to 4 times to ensure uniform composition, and then through the vacuum casting to obtain $\varphi 10 \mathrm{~mm} \times 70 \mathrm{~mm}$ alloy bar. HPT sample is under 5Gpa pressure, $1 \mathrm{R} / \mathrm{min}$, twisted 6 laps, the final size of the sample is $\varphi 20$ $\mathrm{mm} \times 1 \mathrm{~mm}$. 


\subsection{Electrochemical experiments}

The electrochemical corrosion behavior of FeNiCoMoV high entropy alloy before and after HPT was studied in $1 \mathrm{M} \mathrm{NaOH}$ solution. All experiments were carried out at room temperature, all of which were prepared from analytical pure reagents and ultrapure water. For better contrast and reproducibility, all corrosive specimens are polished in accordance with metallographic requirements and cleaned with acetone. Hot air is dry and dried. The macro-corrosion test was carried out using an Ametec VersaSTAT3 potentiometer, all of which were sealed with epoxy resin, leaving the surface of the etched electrode and connected with copper wires. The electrode area of the initial sample is $0.785 \mathrm{~cm} 2$ and the HPT sample area is $1.57 \mathrm{~cm} 2$. Electrochemical tests are used saturated potassium chloride/calomel electrode as the reference electrode, platinum electrode as the auxiliary electrode of the standard three-electrode system [6]. Three kinds of electrochemical detection methods were used for open circuit potential (OCP) test, dynamic potential scanning polarization (PDC) test and electrochemical impedance spectroscopy (EIS) test. The electrochemical impedance spectroscopy [7] has a frequency range of $100 \mathrm{KHz} \sim 10 \mathrm{mHz}$ and an applied excitation voltage of $100 \mathrm{mV}$. The scanning interval of the potentiostatic polarization test is $-1 \mathrm{~V} \sim+1 \mathrm{~V}$ (VSoc) in the $1 \mathrm{M} \mathrm{NaOH}$ solution. , Scanning rate of $1 \mathrm{mV} / \mathrm{s}$. In order to ensure the accuracy of the experiment, each test is repeated three times. In addition, the experimental measured corrosion potential and corrosion current density [8] are realized by Versa-studio software independent Tafel fitting.

\section{Experimental results and analysis}

\subsection{Corrosion behavior of FeNiCoMoV high entropy alloy before and after HPT in $1 \mathrm{M}$ NaOH solution}

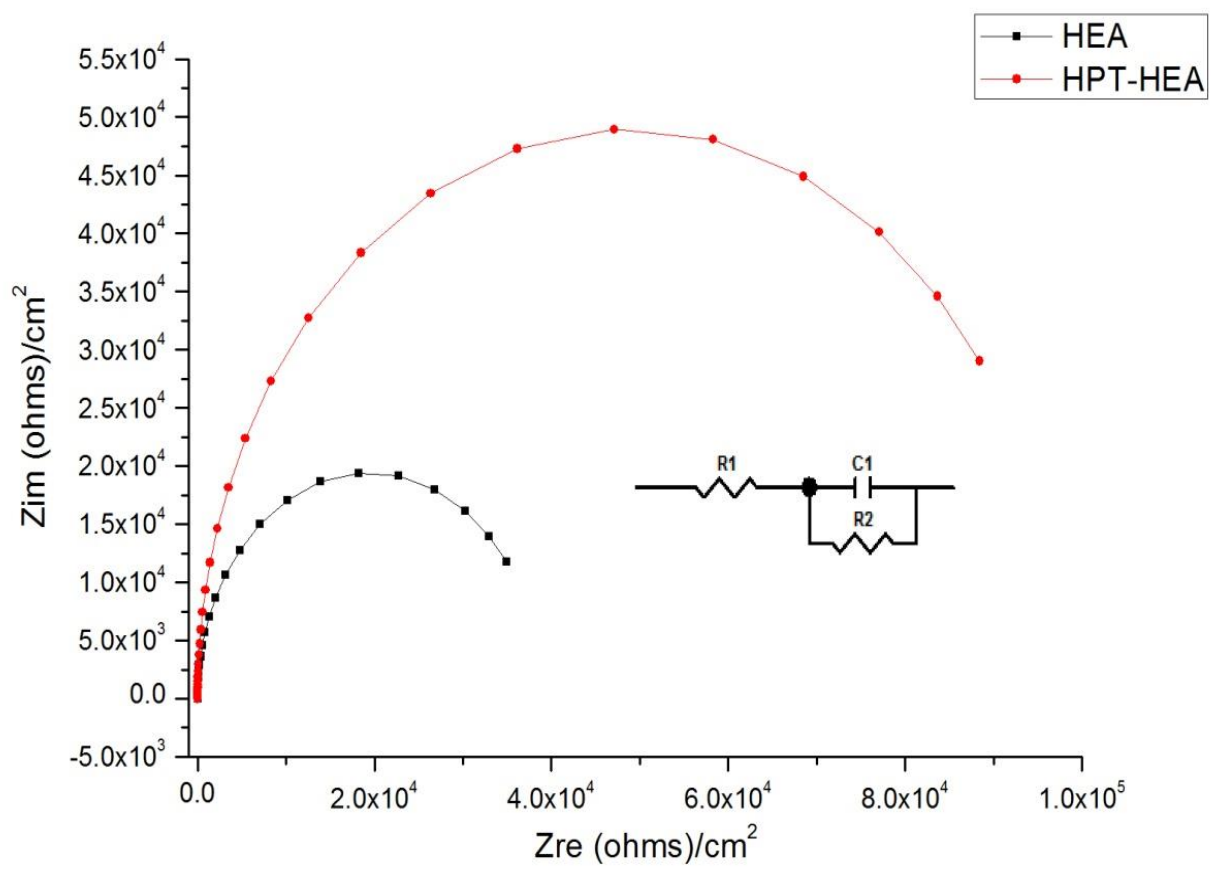

Fig. 1 Electrochemical impedance of the initial state and HPT high entropy alloy in $1 \mathrm{M} \mathrm{NaOH}$ solution

Fig 1 is the initial state and HPT high-entropy alloy in the $1 \mathrm{M} \mathrm{NaOH}$ solution electrochemical impedance diagram, the image by the Zsimpwin software according to the equivalent of the figure made of the circuit, where R1 represents the solution resistance, R2 said charge transfer resistance, $\mathrm{C} 1$ on behalf of the interface capacitance of the sample. It can be seen from the figure, HPT after high entropy alloy sample electrochemical impedance value increased significantly. 


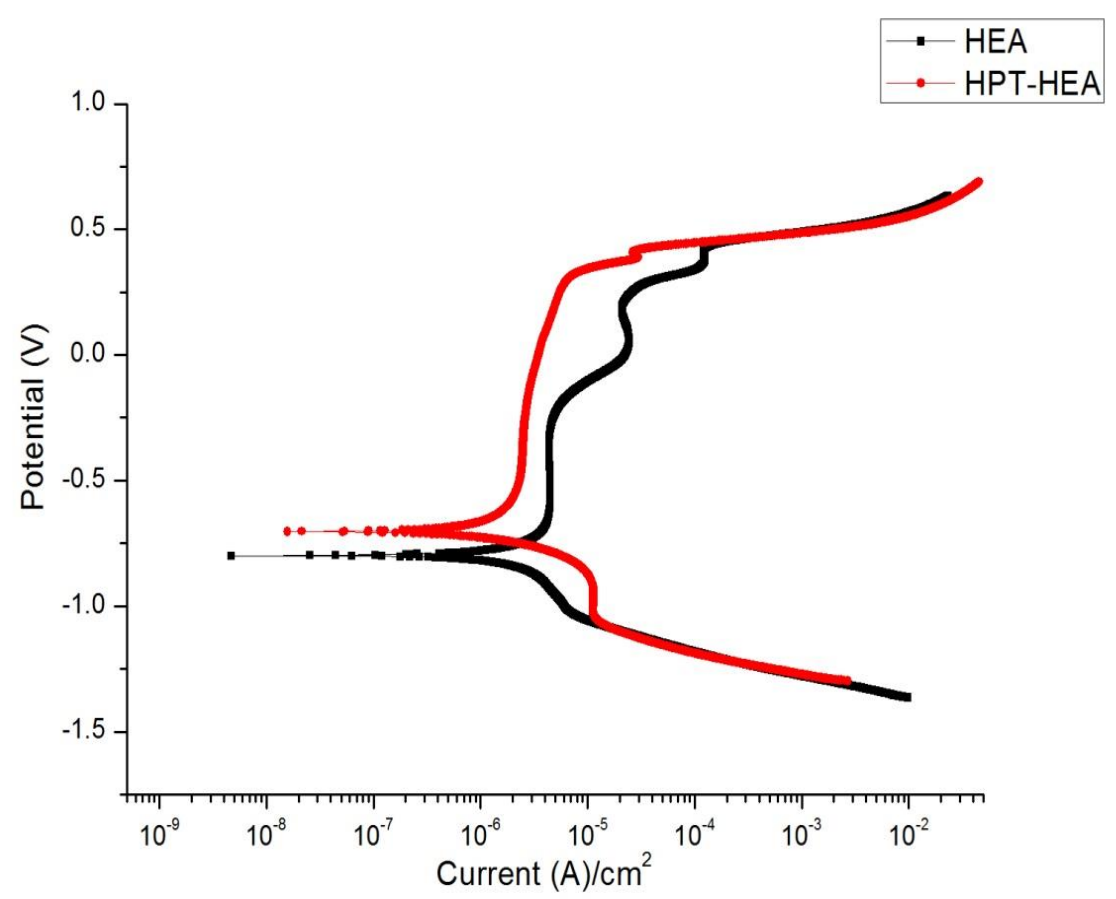

Fig 2. Polarization of the initial state and HPT high entropy alloy in the $1 \mathrm{M} \mathrm{NaOH}$ solution

Table 1. Polarization parameters of the high-entropy alloy before and after HPT in the $1 \mathrm{M} \mathrm{NaOH}$ solution

\begin{tabular}{|c|c|c|c|}
\hline Sample & $\mathrm{E}_{\text {corr }}(\mathrm{mv})$ & $\mathrm{I}_{\text {corr }}\left(\mu \mathrm{A} / \mathrm{cm}^{2}\right)$ & 36.201 \\
\hline HEA & -798.977 & 2.256 & \\
\hline HPT-HEA & -703.302 & & \\
\hline
\end{tabular}

Fig. 2 shows the polarization curves of the initial and HPT high-entropy alloys in the $1 \mathrm{M} \mathrm{NaOH}$ solution. It can be seen that the self-corrosion potential of the HPT state is higher than that of the initial sample, and the corrosion current density is greatly reduced, indicating that the corrosion rate of the sample after HPT is reduced within the active region.

After HPT, the maintain current density of the samples was greatly reduced. The passivation area of the sample is $-600 \sim 300 \mathrm{mV}$, and the passivation zone of the as-cast high entropy alloy is divided into three sections, $-700 \sim-200 \mathrm{mV}, 0 \sim 200 \mathrm{mV}, 350 \sim 400 \mathrm{mV}$, HPT samples showed better passivation. The results show that the corrosion potential of HPT state is $11.97 \%$ higher than that of initial state, 93.77\% lower than that of initial state, and the corrosion resistance is greatly improved.

It can be seen that HPT can effectively enhance the corrosion resistance of high entropy alloy in $1 \mathrm{M}$ $\mathrm{NaOH}$ solution.

\subsection{Corrosion Morphology of FeNiCoMoV High Entropy Alloy in $1 \mathrm{M}$ NaOH Solution before and after HPT}

It can be seen from the fig 3 that after electrochemical corrosion in $1 \mathrm{M} \mathrm{NaOH}$ solution, the corrosion holes are more and large of the initial alloy surface, and the corrosion hole is more serious. The surface of the HPT high-entropy alloy is still smooth and only a small amount tiny pores, which is related to the higher stability of passivation film formed in the surface of HPT state. 




Fig 3. Corrosion morphology of the initial state and HPT high entropy alloy after electrochemical corrosion in the $1 \mathrm{M} \mathrm{NaOH}$ solution

\section{Conclusion}

After the high entropy alloy sample was twisted by 6 turns, the FeNiCoMoV alloy grain showed a gradient distribution, and the phase structure did not change, which was fcc structure. In the $\mathrm{NaOH}$ solution, the electrochemical impedance value of the high entropy alloy after HPT increased significantly, the corrosion potential increased by $11.97 \%$, the corrosion current density was $93.77 \%$ lower than the initial state, and the corrosion resistance was improved obviously.

\section{References}

[1]. Yong Zhang. Microstructures and properties of high-entropy alloys, Progress in Materials Science.2014, 61:1-93.

[2]. Yunjun Zhou Phase Formation and Performance of High Entropy Alloys [D]. [Doctoral Dissertation]. Beijing University of Science and Technology, 2008.

[3]. Wei feng Fang.Study on microstructure and corrosion resistance of CuCrFeNiMn-based high entropy alloy. [D] [Master's Thesis]. Zhengzhou University, 2014

[4]. Qunhua Tang, Pinjiang Dai, Nengbin Hua. Electrochemical Properties of $\mathrm{Al}_{0.3} \mathrm{CoCrFeNi}$ Nanocrystalline High Entropy Alloy in Alkaline Solution [J]. Mechanical Engineering Materials, 2015, 39(12): 1-4

[5]. Huailiang Geng. Study on Microstructure and Properties of Al-Zn-Mg-Cu Alloy by High Voltage Torsion Process [D]. [Master Thesis]. Yanshan University, 2013

[6]. VASILE SOARE, DUMITRU MITRICA, IONUT CONSTANTIN, GABRIELA POPESCU, IOANA CSAKI, MIHAI TARCOLEA, and IOAN CARCEA. The Mechanical and Corrosion Behaviors of As-cast and Re-melted AlCrCuFeMnNi Multi-Component High-Entropy Alloy[J], Metallurgical and Materials Transactions A,2015,46A:1468-1473

[7]. Chunan Cao. Corrosion electrochemical principle [M]. Beijing: Chemical Industry Press, 2008. 122-130

[8]. Y.Qiu, M.A.Gibson, H.L.Fraser and N.Birbilis. Corrosion characteristics of high entropy alloys[J], Materials Science and Technology,2015,31(10)1235-1243 